\title{
Experimental bond behavior of FRP sheets glued on brick masonry
}

Daniel V. Oliveira ${ }^{1}$, Ismael Basilio ${ }^{2}$ and Paulo B. Lourenço ${ }^{3}$

ISISE, Department of Civil Engineering, University of Minho, Guimarães, Portugal

ABSTRACT: This paper deals with the experimental characterization of the mechanical tensile and shear bond behavior of fiber reinforced polymer (FRP) sheets externally glued on masonry prisms, in terms of load capacity and stress distribution along the bonded length. The brick masonry adopted tries to replicate ancient brick masonry, by using handmade low strength solids bricks and low strength lime based mortar. Key parameters relative to the FRP-masonry interface response, particularly bonded length, FRP materials, anchor scheme adopted and shape of masonry substrate were studied. Finally, an analytical bond stress-slip formulation was developed, allowing deducing local bond stress-slip curves directly from the experiments.

KEYWORDS: Brick masonry; Fiber reinforced polymers; Bond; Pull-off; Bond-slip behavior.

${ }^{1} \mathrm{PhD}$, Assistant Professor, ISISE, Department of Civil Engineering, University of Minho, Azurém, P-4800-058 Guimarães, Portugal. Phone: +351 253510 247, fax: +351 253510 217, email: danvco@civil.uminho.pt

${ }^{2} \mathrm{PhD}$, Department of Civil Engineering, University of Minho, Azurém, P-4800-058 Guimarães, Portugal. Phone: +351 253510 247, fax: +351 253510 217, email: ibasilios@gmail.com

${ }^{3} \mathrm{PhD}$, Professor, ISISE, Department of Civil Engineering, University of Minho, Azurém, P-4800-058 Guimarães, Portugal. Phone: +351 253510 209, fax: +351 253510 217, email: pbl@civil.uminho.pt 


\section{INTRODUCTION}

The preservation of cultural heritage buildings is considered a major issue in the cultural life of modern societies. However, this task requires the establishment of proper methodologies, able to tackle simultaneously their considerable architectural and cultural value and their structural safety (ICOMOS 2001). An important topic in current research is the development of efficient and cost-effective strengthening techniques, able to reestablish the performance of cultural heritage buildings and to prevent their brittle failure, particularly under earthquake loading.

In the last years, growing attention has been devoted to the development of innovative materials and advanced technologies to be applied in the preservation of cultural heritage buildings. Strengthening solutions based on the external bonding of fiber reinforced polymer (FRP) composites have become a popular option (e.g. FIB 2001, Bakis et al. 2002, Yuan et al. 2004, Shrive 2006). FRP presents several advantages, as low specific weight, corrosion immunity and high tensile strength. Its flexibility and easy application contribute to a wide range of intervention scenarios. An important research effort has been directed to the characterization of FRP strengthened structures, but the main emphasis has been directed to concrete structures, whereas only few works have dealt with masonry supports, particularly ancient ones. As a consequence, masonry guidelines and recommendations on FRP strengthening reflect this unbalanced situation, being the theoretical framework

usually derived from research on concrete, as testifies the recent Italian guidelines for FRP strengthening of masonry (CNR-DT200 2004). Further research encompassing the specific features of masonry is therefore needed.

The success of externally bonded strengthening strategies depends to a great extent on the capacity of the FRP-masonry interface in developing an efficient load transfer mechanism. The occurrence of debonding along an interface is the direct cause of failure in a great number of masonry supports. This feature demands a comprehensive characterization of the mechanical behavior of FRP-masonry interfaces. Only recently the characterization of externally bonded masonry substrates with composites has been investigated (Cosenza et al. 2000, Accardi and La Mendola 2004, Aiello and Sciolti 2006, Grande et al., 2008, Panizza et al. 2008), involving the analysis of different key parameters as substrate materials, composite systems, bonded length, test setup, among others. In spite of the differences among researchers, two aspects seem to be common: use of single units and adoption of double-shear test configurations. In addition, authors tend to use carbon fibers. 
Different test setups have been adopted for the characterization of bond behavior between FRP composites and concrete-based substrates, but a unique well-established setup has not been defined yet. Existing test setups are usually classified as: single-shear tests (Täljsten 1997), double-shear tests (Lee et al. 1999) and beam tests (De Lorenzis et al. 2001). A comprehensive review of available test setups is given in Chen et al. (2001). In spite of the simplicity associated to double-shear test configurations, the geometric symmetry imposed to the FRP strengthening simultaneously in two substrates cannot assure an equally distributed load is applied throughout the test, due to the different intrinsic nonlinear behavior of the substrate. This might lead to difficulties in interpreting the results. From the three possible configurations given above, the single-shear test setup is closer to real applications, thus being considering the most reliable setup to be used in research (Mazzotti et al. 2009).

This paper is focused on the experimental bond behavior of FRP-masonry interfaces, considering both tensile and shear bond. Monotonic pull-off and single-shear tests were carried out under displacement control. Pull-off tests provide valuable information about failure under mode I, whereas an existing single-shear test setup (Täljsten 1997) was adapted to be used for the experimental bond behavior characterization (mode II). Within the framework of thin externally bonded plates to rigid substrates, mode I behavior is associated to the plate-substrate interface deformation under normal tensile stresses, while mode II behavior corresponds to the interface deformation under shear stresses.

Two types of handmade clay bricks were used for pull-off. Different bond lengths, composite materials, anchor schemes and support shapes were considered in the single-shear experiments. Masonry prisms were used instead of single units to include the effect of mortar joints and also to have specimens representative of real applications. Glass FRP composites (GFRP) were chosen as the main strengthening material. When compared with carbon fibers, the lower axial stiffness of GFRP seem more appropriate to use for low strength and low stiffness masonry structures, reducing stress concentrations along the bonded length.

The main objective of the present paper is the characterization of the ultimate load and failure patterns of masonry prisms strengthened with FRP materials under mode I and mode II. In addition, the stress profile along the bonded length was characterized for the different arrangements and the local bond stress-slip curves were analytically derived from experiments and discussed. 


\section{MATERIALS CHARACTERIZATION}

FRP sheets were externally bonded to masonry prisms following the typical wet layup system, namely the application of an epoxy primer on the masonry substrate followed by the application of putty to level the masonry surface and finally the application of epoxy resin and a single layer fiber sheet oriented perpendicularly to mortar joints. The mechanic characterization of all components is briefly described next, with further details provided in Basilio (2007).

\section{Bonding materials}

The computation of Young's modulus, tensile strength and strain at failure of both primer and resin was based on molded dog-bone shaped specimens, prepared according to guideline ISO $527-2$ (1993). Putty specimens were also prepared according to the same guideline. However, due to its relatively high viscosity, the preparation of putty specimens required a more delicate procedure. Following specimen molding and drying, a strain gauge was glued at the middle of the gauge length and aligned with the longest specimen’s symmetrical axis. The tensile testing of all specimens was carried out by means of a Universal Zwick machine with a load cell capacity of $5 \mathrm{kN}$, in accordance with guidelines ISO 527-1 (1993) and ISO 527-2 (1993). All primer and putty specimens failed at the mid-gauge length, the ideal rupture mode. In turn, resin specimens failed close to the grip, but all failure sections were located inside the gauge length.

Table 1 presents the tensile strength and strain at failure as well as the Young's modulus values of the three bonding materials. Each value was obtained as the average value of five experimental results. The same results are graphically illustrated in Figure 1(a) in terms of stress-strain diagrams. The results obtained exhibit low coefficients of variation, which seems to indicate satisfactory molding and testing procedures.

The three bonding materials exhibited the typical very fragile behavior and, therefore, no post-peak behavior was captured. While primer and putty specimens present a clear linear elastic behavior, resin specimens seem to possess an incipient non-linear behavior. Similar experimental data range values of tensile strength and Young's modulus were reported for primer and resin by Casareto et al. (2002) and for the putty by Bonaldo et al. (2005).

\section{Fibers}

The mechanical characterization of the bond behavior through single-shear tests, to be described latter on, was performed using a unidirectional carbon fiber fabric (reference CF-120, mass density of $0.20 \mathrm{~kg} / \mathrm{m}^{2}$ ) and a bidirectional glass fiber fabric (reference G-AR 90/10, mass density of $0.44 \mathrm{~kg} / \mathrm{m}^{2}$ ), with $90 \%$ of the fibers 
oriented in the main direction. The purpose of using both fibers was to understand the potential influence of the FRP material on the bond behavior.

All specimens were prepared and tested according to the guideline ISO 527-5 (1997). The fibers were wrapped with a tiny layer of resin, and uniformly brushed on both sides. After cutting, specimens were mounted and tested in a universal Instron testing machine with a load cell capacity of $50 \mathrm{kN}$. Recommendations of good practice provided by the manufacturer were followed during the entire test procedure. Results of tensile strength, strain at failure and Young's modulus are displayed in Table 1 (average values from 5 specimens), while the tensile stress-strain curves are depicted in Figure 1(b), for both glass and carbon fibers.

The tensile behavior is characterized by an almost linear stress-strain pre-peak relationship. Even though both materials failed to exhibit a post-peak branch, carbon fibers had a sudden and explosive failure when compared with the smoother misaligned thread of glass fibers at collapse. Figure 1(b) shows clearly that the lower stiffness and larger failure strain exhibited by glass fibers makes them a better option from the mechanical point of view to be used in low strength and low stiffness masonry structures.

\section{Masonry}

Aiming at replicating ancient brick masonry structures, two different Portuguese traditional solid clay bricks and a commercial pre-mixed hydraulic lime-based mortar (Mape-antique MC) were selected to construct the masonry prisms to be strengthened with composite materials. The two types of bricks, here termed as brick type 1 and brick type 2, have different geographical origins and sizes. The resulting masonry prisms were prepared with the same mortar and are termed here as masonry type 1 (MT1) and masonry type 2 (MT2). A detailed description on the geographical origin can be found in Basilio (2007).

Tests performed on eight cylindrical mortar specimens with dimensions $\varnothing 50 \times 110 \mathrm{~mm}^{2}$ provided an average compressive strength of $7.3 \mathrm{~N} / \mathrm{mm}^{2}$ and a coefficient of variation $(\mathrm{CoV})$ of $8 \%$. The compressive strength and the Young's modulus of masonry were assessed trough the testing of 10 representative prisms of each masonry type, after two weeks of curing. The dimensions of the prisms reached $235 \times 130 \times 90 \mathrm{~mm}^{3}$ (four bricks of 45 $50 \mathrm{~mm}$ thickness and three mortar joints of approximately 10-15 mm thickness) for masonry type 1 (MT1), and $163 \times 100 \times 50 \mathrm{~mm}^{3}$ (five bricks of $25 \mathrm{~mm}$ thickness and four mortar joints of $10 \mathrm{~mm}$ thickness) for masonry type 2 (MT2). All tests were performed under monotonic displacement control, allowing the characterization of the softening behavior. For masonry type 1 an average compressive strength of $8.8 \mathrm{~N} / \mathrm{mm}^{2}(\mathrm{CoV}=27 \%)$ and an average elastic modulus of $1300 \mathrm{~N} / \mathrm{mm}^{2}(\mathrm{CoV}=39 \%)$ were obtained, whereas for masonry type 2 an average 
compressive strength of $9.1 \mathrm{~N} / \mathrm{mm}^{2}(\mathrm{CoV}=16 \%)$ and an average elastic modulus of $2040 \mathrm{~N} / \mathrm{mm}^{2}(\mathrm{CoV}=34 \%)$ were computed. Despite the moderate to high CoV's found and the different geographic origins of bricks, the compressive strength of both masonry types can be considered similar.

\section{PULL-OFF TESTS}

The failure of externally bonded quasi-brittle substrates (as masonry) with FRP sheets is generally controlled by the interfacial shear and normal stress distributions (De Lorenzis and Zavarise 2008). It is here assumed that the tensile failure of the FRP sheets is reached for loads much higher than of the interface. For joints subjected to predominant shear stresses, debonding takes place (mode II failure). However, in the proximity of inclined cracks or curved substrates, a mixed-mode condition occurs, where the interfacial normal strength may play an important role in the definition of the collapse load (De Lorenzis and Zavarise 2008). By means of the standard pull-off test applied to FRP-masonry interfaces, it is possible to obtain a good estimation of its tensile strength, under mode I failure.

\section{Test setup and test procedure}

For the pull-off tests, two types of masonry (MT1 and MT2) and two types of FRP fibers (carbon and glass) were used. For each combination of masonry and FRP types, five specimens were tested. Following the cleaning of the masonry substrate, the composite system was applied according to the wet layup system. After primer application, a FRP strip of $70 \mathrm{~mm}$ width was glued on masonry. Afterwards, a partial-depth core with $49 \mathrm{~mm}$ diameter and $10 \mathrm{~mm}$ depth was drilled, see Figure 2, leading to the creation of circular strengthened areas where rigid steel plates were glued. The drillings were centered with the masonry joints. The area of the masonry cylinder to be tested under direct tensile load was composed, on average terms, of 2/3 of brick and 1/3 of mortar. The adopted location of the cylinders is justified by the need of involving the mortar on the interface load capacity assessment.

The tests were carried out using a Proceq DYNA Z15 portable machine. A constant velocity rate of $20 \mathrm{kPa} / \mathrm{s}$ was applied so the ultimate load was attained in about one minute. These tests were performed following the guideline ASTM D4541-02 (2002).

\section{Normal tensile strength}

Table 2 illustrates the average pull-off test results for the four combinations of masonry and FRP types. The results indicate that the pull-off strength is practically independent of the FRP material and masonry type, despite 
of the moderate coefficient of variation. This feature can be explained based on the failure pattern registered. For all specimens tested, failure was characterized by the ripping of a thin layer of brick and mortar (peeling). The rigid plate surface was fully covered of brick and mortar, which exhibited an irregular thickness variation. The results obtained show that the tensile strength of the interfaces tested depends on the tensile strength of the substrate, which is the weakest element of the FRP-resin-masonry assemblage under direct tensile loading (mode I), and not on the tensile strength of the bonding agents. These results seem to indicate that for low strength substrates strengthened as described above, the tensile strength of the substrate is much lower than of the bonding agents, see also Table 1 . Within this context, the use of a cheaper and low strength bonding agent seems to be advantageous.

This result shows also that the two types of bricks used possess a similar tensile strength, despite the differences in terms of geographical origin and production procedures, which corroborate the conclusion about the compressive strength given above.

\section{EXPERIMENTAL BOND BEHAVIOR CHARACTERIZATION}

\section{Test setup and test procedure}

The masonry prisms with dimensions $235 \times 130 \times 90 \mathrm{~mm}^{3}$ were prepared and strengthened according to the techniques described above. Given the mechanical resemblance of the two masonry types used for the pull-off tests, only masonry prisms type 1 were used here.

The prisms were strengthened with a glass FRP strip of $25 \mathrm{~mm}$ width and $150 \mathrm{~mm}$ length. This specimen was taken as a reference from which several variations were adopted. To evaluate the influence of the major parameters that dominate the bond behavior of the FRP-masonry interface, the following variations with respect to the reference specimen were considered, see also Figure 3:

- Bonded length;

- FRP material;

- Use of anchor schemes;

- $\quad$ Shape of substrate.

Therefore, eight different types of arrangements were tested, as illustrated in Figure 3, being termed according to the following description:

- G150RS: reference or standard specimen, with a flat surface and strengthened with a 25 mm width and 150 mm length glass FRP strip. No anchor devices were used; 
- G100RS: The difference between this specimen and the previous one is only related to the bonded length, being now $100 \mathrm{~mm}$;

- G200RS: A $200 \mathrm{~mm}$ bond length was considered in this arrangement with respect to the reference specimen;

- C150RS: This specimen is equal to the reference one except for the composite material, made now of carbon fibers instead;

- G150RI: In this specimen a non-conventional anchor scheme based on the use of flexible GFRP-based spike anchors was implemented (Casareto et al. 2002). Glass fibers impregnated with resin were inserted into a predrilled $6 \mathrm{~mm}$ diameter hole, close to the free unloaded end, and fanned out over the strip. Only one spike anchor was considered per specimen.

- G150RT: Another non-conventional anchor scheme was tested on this specimen. A FRP bar of $45 \mathrm{~mm}$ length and $6 \mathrm{~mm}$ diameter was placed transversally over the strip, close to the free end, and glued to the strip and to the substrate. A groove of about $8 \mathrm{~mm}$ diameter was previously made on the brick in order to accommodate the bar, which was intended to mechanically anchor the strip as to increase the ultimate load.

- G150ES: In this specimen a concave surface shape $(r=750 \mathrm{~mm})$ was adopted instead, aiming at representing the intrados surface of masonry arches and vaults, keeping constant the remaining parameters with regard the reference specimen G150RS;

- G150XS: For this specimen a convex surface shape $(r=750 \mathrm{~mm})$ was considered for the application of the GFRP strip, to replicate the extrados conditions of masonry arches and vaults. All the remaining parameters were kept unchanged with respect the reference specimen.

The steel device used to test the prisms was composed of an $\mathrm{H}$ profile welded to a rigid plate and stiffened by means of two welded lateral bars, see Figure 4. The prisms were positioned on the steel device and firmly clamped to it as to avoid any type of rigid body movement, while the device was mounted on the Universal Instron testing machine aforementioned, able to work under axial displacement or load control. All masonry surfaces in contact with the steel device were carefully smoothed to minimize any non-uniform stresses distribution, thus leading to an enhanced clamping solution. The tests were carried out under displacement control, by imposing a constant displacement rate of $0.1 \mathrm{~mm} / \mathrm{min}$ at the loaded end of the FRP strip, while the resulting load was measured by means of a load cell. All tests were carried out up to failure. In total, 5 specimens of each series were tested, resulting in 40 specimens successfully tested. 
The relative displacements between the FRP and the substrate were measured at the loaded (LVDT1) and free ends (LVDT2), see Figure 4b. Any possible rigid body movement of the steel device was also monitored (LVDT3). For each specimen, two strain gauges were used at the thirds of the bonded length, see also Figure 4. By assuming that strain is constant in the composite cross section and that the FRP performs in linear elastic regime, it is possible to know the FRP tensile stress at four points along the bonded length.

\section{Ultimate load}

Table 3 presents the tensile stress applied to FRP that caused failure of the strengthening, in terms of average and coefficient of variation values. The results obtained show that by decreasing the bond length from $150 \mathrm{~mm}$ (G150RS) to $100 \mathrm{~mm}$ (G100RS) a lower ultimate load is reached, as expected, resulting in an average decrease of $28 \%$. In turn, when shifting from $150 \mathrm{~mm}$ to $200 \mathrm{~mm}$ (G200RS), the increment in load capacity is relatively small, about just 3\%. These results are due to the change of failure mode. For the bond length of $100 \mathrm{~mm}$, all specimens failed due to debonding of the FRP with the detachment of a thin layer of brick and mortar, as mentioned in previous researches (e.g. Aiello and Sciolti 2006). For the case of $150 \mathrm{~mm}$ bond length, one of the five specimens failed due to tensile rupture of the FRP strip. Furthermore, for the case of $200 \mathrm{~mm}$ bond length, three specimens out of five failed due to tensile rupture of FRP strip, whereas the average failure stress of G200RS series (Table 3) represents 90\% of the average tensile strength of glass fibers (Table 1). Most probably, further increases of the bond length would lead to marginal increments of the load capacity. In conclusion, the results seem to show that the $150 \mathrm{~mm}$ bond length is close to the optimal bond length, meaning that further increases of this length do not produce noticeable increments of the ultimate load.

The results described above suggest that the use of a stronger FRP material would allow an increase of the ultimate load. Indeed, the experimental results confirm this hypothesis. The use of carbon fibers (C150RS) instead of glass ones (G150RS) allowed an increase of the ultimate load of about 16\%, as shown in Table 3 . Due to the higher tensile strength of the carbon fibers, the tensile failure was prevented and thus all specimens failed by debonding, making clear that the optimal bond length increases if stronger fibers are used.

As for the anchor schemes tested, the effect of a handmade flexible spike placed close to the free end (G150RI) was noticeable. The anchorage effect promoted by the spike shifted the failure mode from debonding to tensile failure. As a result, the average ultimate load registered an increase of about $26 \%$. The second anchor scheme (G150RT), composed by a FRP bar placed transversally over the strip and glued to it and to the substrate, did not seem to be effective as only a marginal increase of $3 \%$ of the average ultimate load was 
registered. This means that the adopted arrangement was not able to provide an enhanced anchoring effect. In line with this result, failure was due to debonding of the FRP strip.

The consideration of curved substrates introduces normal stresses along the FRP-masonry interface due to equilibrium, either of tensile (concave substrates) or compressive (convex substrates) nature. In particular, it was expected that the interaction between the normal tensile and shear stresses (at the concave interfaces) leaded to a reduction of the ultimate load, as numerically foreseen (De Lorenzis and Zavarise 2009). The failure of the specimens with curved substrates was due to debonding, with no visual differences with respect to flat substrates. The experiments showed a 4\% decrease of the average strength of concave specimens (G150ES), see Table 3. This reduced variation in strength might suggest a marginal influence of normal stresses in failure. However, it is possible that the influence of normal stresses in the debonding process might increase for smaller radii of curvature, thus implying larger reductions in strength. As for the convex specimens, results showed a decrease of $13 \%$ in comparison with the reference series. This was an unexpected result, as a decrease in load was not foreseen. Further similar experiments are planned to better understand the influence of curvature.

The coefficients of variation (CoV) range between $6 \%$ and $20 \%$, which seems to be slightly high considering the laboratory conditions used, even if moderate to high CoV values are usually associated with masonry. However, other authors have reported even higher CoV values (e.g. Aiello et al. 2007). It is possible that the presence of mortar joints might constitute an additional source of scattering with regard to the use of single units.

In general, the specimen's failure was due to debonding of the FRP strip, see Figure 5, except for the cases where tensile failure of the FRP took place (as addressed above). In both failure modes a brittle behavior was registered. In the case of tensile failure, this was due to the well-known brittleness of the FRP, which has no post-peak, see also Figure 1(b). For the general case, the brittle behavior of the specimens was due to the brittleness of the brick (Basilio 2007) combined with the inevitable release of elastic strain energy stored in the specimen during the loading process. Preliminary tests using displacement control options were attempted, but it was not possible to avoid uncontrolled failure of specimens due to debonding.

\section{Load transfer}

The load transfer phenomenon between the tensile FRP strip and the brick masonry can be followed in Figure 6 to Figure 9, where the average stress profiles at the FRP strip are shown for 25\%, 50\%, 75\% and 100\% of the ultimate load. The stress was computed based on the strain measurements and assuming linear elastic behavior for the FRP. The depicted lines represent linear interpolations between mean values of five specimens 
tested within each series. As explained previously, it became impossible to register the stress profile during the softening regime.

The normal stress increases from the free to the loaded end and with the applied load, in every cross section (except for the free end, where it is assumed to be equal to zero), but its variation depends on specimen's series. In the same way, the shape of the stress profile depends on the series. For low load levels, the stress profile is essentially exponential, for all specimens, indicating that the load transfer occurs along a short length close to the loaded end. With the increase of load level a longer load transfer length is mobilized. In most of the specimens, the initial exponential stress profile was progressively substituted by a quasi triangular one, see Figure 6 to Figure 9. Analogue results with the same trend have been reported by other authors for concrete (e.g. Bizindavyi and Neale 1999) and masonry (e.g. Aiello and Sciolti 2006). The exceptions are the C150RS series, where a kind of extended S-shape profile was observed, and the G200RS series, where the stress profile is kept exponential along the entire load procedure. Most probably, the "premature" tensile failure of the FRP strip in the G200RS series (in the sense that the interface was able to sustain further load) did not allow the evolution of the stress profile. Apparently, the anchor schemes used did not have a visible influence on the stress profile, inducing almost triangular stress profiles at the peak load. As for the concave and convex surface shapes, it seems that the stress profile tends to exhibit a slightly convex shape at the ultimate load (see also Figure 9).

\section{ANALYTICAL APPROACH}

The equilibrium of a piece of FRP strip of length $d x$ bonded to masonry is expressed according to:

$$
d \sigma_{F R P}(x) w_{F R P} t_{F R P}=\tau(x) w_{F R P} d x
$$

where $\sigma_{F R P}$ is the normal stress at the FRP strip, $\tau$ is the bond stress at the FRP-masonry interface, $w_{F R P}$ and $t_{F R P}$ are the width and the thickness of the FRP strip, respectively, and $x$ is the longitudinal coordinate, with origin at the free end. Assuming linear elastic behavior of the composite, eq. (1) can be rewritten as

$$
\tau(x)=E_{F R P} t_{F R P} \frac{d \varepsilon_{F R P}(x)}{d x}
$$

where $E_{F R P}$ and $\varepsilon_{F R P}$ are the Young's modulus and the normal strain of the FRP strip, respectively. By assuming that the deformability of the epoxy adhesive and masonry are negligible when compared with the FRP deformability, $\varepsilon_{F R P}$ can be expressed simply as

$$
\varepsilon_{F R P}(x)=\frac{d s(x)}{d x}
$$


where $s$ is the slip between masonry and the reinforcement. Integrating eq. (3) and neglecting the slip value at the free end (confirmed by LVDT2 readings), the slip at a distance $x$ from the free end reads

$$
S(x)=\int_{0}^{x} \varepsilon_{F R P}(x) d x
$$

By considering the available discrete strain readings $\varepsilon_{F R P}\left(x_{i}\right)$ along the bonded length, $s(x)$ and $\tau(x)$ can be approximated numerically, by integration of eq. (4) and differentiation of eq. (2), as follows

$$
\begin{aligned}
& s\left(x_{i}\right)=\frac{1}{2} \sum_{k=1}^{i}\left(\varepsilon_{F R P}\left(x_{k}\right)+\varepsilon_{F R P}\left(x_{k-1}\right)\right)\left(x_{k}-x_{k-1}\right) \\
& \tau\left(x_{i}\right)=\frac{1}{2} E_{F R P} t_{F R P}\left(\frac{\varepsilon_{F R P}\left(x_{i}\right)-\varepsilon_{F R P}\left(x_{i-1}\right)}{x_{i}-x_{i-1}}+\frac{\varepsilon_{F R P}\left(x_{i+1}\right)-\varepsilon_{F R P}\left(x_{i}\right)}{x_{i+1}-x_{i}}\right)
\end{aligned}
$$

Analytical curves might show a certain degree of irregularity, as they are based on discrete and limited strain readings. Whereas numerical integration is a smoothing process, numerical differentiation tends to magnify irregularities. In order to smooth the analytical curves, additional discrete "readings” were considered between each 2 consecutive real strain records, as average values of adjacent readings. As an example, Figure 10 shows envelopes of analytical bond stress profiles for G150RS series, considering $50 \%$ and $100 \%$ of the ultimate load.

\section{Local bond stress-slip relationship}

The local bond stress-slip relationship $\tau(s)$ can be generated by combining the individual $s(x)$ and $\tau(x)$ curves, obtained numerically, and drawn for each $x$ coordinate along the bonded length. Diagrams from Figure 11 to Figure 14 represent the interfacial local bond-slip curves derived for all the series, computed at the loaded end, so a larger extension can be observed. In spite of the differences among series and among specimens within the same series, as well as errors due to numerical differentiation, a general trend might be observed. For low loads, bond-slip curves show an initial linear elastic stage characterized by similar stiffness within each series. With increasing loading, a hardening stage is originated at around $60 \%-80 \%$ of the ultimate load. The extension of this hardening branch is variable, ranging from an incipient and short nonlinear behavior to a progressive nonlinear behavior. Although differences exist among series, a trend is visible within each one.

In some specimens it is possible to identify a ductile phase in the sense that specimens can sustain a constant bond stress with increasing slip. The next stage is characterized by a marked softening behavior, although this stage was not registered in all specimens. Available results are not totally conclusive about the existence of 
residual bond strength after debonding, due to friction over the debonded length. While some specimens indicate the absence of residual bond strength, others suggest its existence.

\section{CONCLUDING REMARKS}

The experimental performance of FRP strengthened clay masonry prisms under tensile and shear loading was presented and discussed in the paper. The results from pull-off tests show that failure was due to the ripping of a thin layer of brick and mortar, making the substrate the weakest element (lower tensile strength) of the FRPresin-masonry assemblage under mode I. Therefore, the tensile strength of the brick and mortar controls the tensile strength of the FRP-masonry interface. Within this context, it seems reasonable to use low strength bonding agent in the strengthening of (low strength) masonry constructions.

The increase of the bonded length allowed shifting from debonding to tensile failure, with location of the optimal bond length. It was observed that the value of this parameter depends on the FRP tensile strength. Debonding of the FRP involved always the detachment of a thin layer of brick and mortar. The use of a simple handmade spike placed at the free end was very effective, allowing an increase of the average ultimate load of about $26 \%$. This result shows that suitable anchorage schemes might be effective in preventing or delaying debonding phenomena in masonry structures. The use of concave substrates caused a slight decrease of the ultimate load. This was an expected result, however the experimental behavior of curved substrates (including the variation of the radius of curvature) requires more attention from researchers as available experimental information is almost absent from literature.

The analysis of the stress profiles in bond specimens allowed understanding how stress varies along the bonded length and between load increments. For low load levels, the stress profile is exponential, showing that load transfer occurs along a short length close to the loaded end. With the increase of the load level, a longer load transfer length is mobilized and the initial exponential stress profile was progressively substituted by a quasi triangular one.

The development of an analytical approach supported on the experiments allowed drawing the local bondslip curves. These curves show an initial linear elastic stage followed by a hardening stage at around $60 \%-80 \%$ of the ultimate load. In some specimens it was possible to identify a ductile stage. The next stage was characterized by a noticeable softening behavior, although this stage was not registered in all specimens. Available results are not conclusive about the existence of residual bond strength after debonding. 
The local bond stress-slip curves seem somehow to be different from the ones derived from externally reinforced concrete specimens (e.g. see Yuan et al. 2004), so further research should be developed on this issue, as bond stress-slip laws suitable for masonry are necessary. Another relevant feature is the need for a suitable test setup configuration for masonry bond tests, probably based on a single-shear test setup, able to provide an efficient characterization of the softening regime.

\section{ACKNOWLEDGMENTS}

The financial support provided by the Portuguese Science and Technology Foundation through the POCTI/ECM/38071/2001 project is acknowledged. FRP composites and pre-mixed mortar used in the laboratory tests were gently offered by BETTOR MBT and MAPEI, respectively, to whom authors are grateful. The second author would like to express his gratitude to CONACYT for the scholarship granted.

\section{REFERENCES}

Accardi, M., La Mendola, L. (2004). "Stress transfer at the interface of bonded joints between FRP and calcarenite natural stone”, IV International Seminar of Structural Analysis of Historical Constructions, Padova, Italy, 867-874.

Aiello, M.A., Sciolti, M.S., (2006). "Bond analysis of masonry structures strengthened with CFRP sheets”, Construction and Building Materials, 20, 90-100.

Aiello, M.A., Sciolti, M.S., Triantafillou, T C. (2007). “FRP-strengthened masonry: bond aspects”, FRPRCS-8, University of Patras, Patras, Greece, CD-ROM, 9 p.

ASTM D4541-02 (2002). "Standard test method for pull-off strength of coatings using portable adhesion testers”.

Bakis, C.E., Bank, L.C., Brown, V.L., Cosenza, E., Davalos, J.F., Lesko, J.J., Machida, A., Rizkalla, S.H., Triantafillou, T.C. (2002). "Fiber-reinforced polymer composites for construction-state-of- the-art review", J. Compos. for Constr., 6(2):73-87.

Basilio, I. (2007). "Strengthening of arched masonry structures with composites materials", PhD Thesis, Universidade do Minho, Portugal. Available at www.civil.uminho.pt/masonry.

Bizindavyi, L., Neale, W. (1999). “Transfer lengths and bond strength for composites bonded to concrete”, J. Compos. for Constr., 3(4), 153-160. 
Bonaldo, E., Barros, J. O., Lourenço, P. B. (2005). "Bond characterization between concrete substrate and repairing SFRC using pull-off testing”. Int. J. Adhesion \& Adhesives, 25, 463-474.

Casareto, M., Oliveri, A., Romelli, A. (2002). “Strengthening of masonry: Opportunities and challenges in the use of composites”. MSc thesis, University of Genoa and University of Missouri Rolla.

Cosenza, E., Manfredi, G., Occhiuzzi, A. and Pecce, M.R. (2000). "Toward the investigation of the interface behaviour between tuff masonry and FRP fabrics”, Mechanics of masonry structures strengthened with FRP materials, Venice, Italy.

Chen, J., Yang, Z., Holt, G. (2001). "FRP or steel plate to concrete bonded joints: Effects of test methods on experimental bond strength”, Steel and Composites Structures, 1(2), 231-244.

CNR-DT200 (2004). “Guide for the design and construction of externally bonded FRP systems for strengthening existing structures.” National Research Council of Italy, Rome, Italy.

De Lorenzis, L., Miller, B., Nanni, A. (2001). “Bond of FRP laminates to concrete.ACI Materials Journal”, 98(3), 256-264.

De Lorenzis, L., Zavarise, G. (2008). “Modeling of mixed-mode debonding in the peel test applied to superficial reinforcements”. Int. J. Solids and Structures, 45, 5419-5436.

De Lorenzis, L., Zavarise, G. (2009). “Interfacial stress analysis and prediction of debonding for a thin plate bonded to a curved substrate”, Int. J. Non-Linear Mechanics, 44(4), 358-370.

FIB Bulletin No.14. Externally bonded FRP reinforcement for RC structures 2001; Lausanne, Switzerland.

Grande, E. Imbimbo, M., Sacco, E. (2008). "FRP-strengthening of masonry structures: Effect of debonding phenomenon”, VI International Conference on Structural Analysis of Historical Constructions, Bath, 10171023.

ICOMOS (2001). "Recommendations for the analysis, conservation and structural restoration of architectural heritage”, International Scientific Committee for Analysis and Restoration of Structures of Architectural Heritage, Paris.

ISO 527-1 (1993). "Plastics: Determination of tensile properties. Part 1: General Principles”, International organization for standardization. 
ISO 527-2 (1993). "Plastics: Determination of tensile properties. Part 2: Test conditions for molding and extrusion plastics”, International organization for standardization.

ISO 527-5 (1997). "Plastics determination of tensile properties, Part 5: Test conditions for unidirectional fibrereinforced plastics composites”, International organization for standardization.

Lee, Y.J., Boothby, T.E., Bakis. C.E., Nanni. A. (1999). "Slip modulus of FRP sheets bonded to concrete”. J. Compos. for Constr., 3(4), 161-167.

Mazzotti, C., Savoia, M., Ferracuti, B. (2009). “A new single-shear setup for stable debonding of FRP-concrete joints”, Const Building Materials, 23, 1529-1537.

Panizza, M., Garbin, E., Valluzzi, M.R., Modena, C. (2008). “Bond behaviour of CFRP and GFRP laminates on brick masonry”, VI International Conference on Structural Analysis of Historical Constructions, Bath, 763770.

Shrive, N.G. (2006). "The use of fibre reinforced polymers to improve seismic resistance of masonry", Construction and Building Materials, 20, 269-277.

Täljsten, B. (1997). “Defining anchor lengths of steel and CFRP plates bonded to concrete”, Int. J. Adhesion and Adhesives 17(4), 319-327.

Yuan H, Teng JG, Seracino R. (2004). “Full-range behavior of FRP to concrete bonded joints”, Engineering Structures, 26, 553-565. 


\section{LIST OF TABLES CAPTIONS}

Table 1 - Tensile parameters and Young's modulus of the bonding materials and of the fiber reinforced polymers employed (average value of five specimens; the coefficient of variation is provided inside parentheses).

Table 2 - Pull-off test results (average value of five specimens; the coefficient of variation is provided inside parentheses).

Table 3 - Normal strength of the eight different bond arrangements. 
Table 1 - Tensile parameters and Young's modulus of the bonding materials and of the fiber reinforced polymers employed (average value of five specimens; the coefficient of variation is provided inside parentheses).

\begin{tabular}{|c|c|c|c|}
\hline \multirow{2}{*}{ Material } & \multicolumn{2}{|c|}{ Tensile parameters } & \multirow{2}{*}{$\begin{array}{c}\text { Young's modulus } \\
{\left[\mathrm{kN} / \mathrm{mm}^{2}\right]}\end{array}$} \\
\hline & Stress $\left[\mathrm{N} / \mathrm{mm}^{2}\right]$ & Strain [\%] & \\
\hline Primer & $5.9(3 \%)$ & $4.76(12 \%)$ & 0.32 (10\%) \\
\hline Putty & 32.2 (3\%) & $0.44(7 \%)$ & 7.44 (2\%) \\
\hline Resin & 48.6 (9\%) & 1.91 (16\%) & 3.67 (8\%) \\
\hline Glass fibers & 1473 (15\%) & 3.66 (12\%) & 80.2 (4\%) \\
\hline Carbon fibers & 2535 (5\%) & 2.42 (9\%) & $215.6(2 \%)$ \\
\hline
\end{tabular}


Table 2 - Pull-off test results (average value of five specimens; the coefficient of variation is provided inside parentheses).

\begin{tabular}{ccc}
\hline Masonry type & Fiber type & $\begin{array}{c}\text { Tensile strength } \\
{\left[\mathrm{N} / \mathrm{mm}^{2}\right]}\end{array}$ \\
\hline MT1 & Glass & $1.1(18 \%)$ \\
MT2 & Carbon & $1.2(27 \%)$ \\
& Glass & $1.2(20 \%)$ \\
\hline
\end{tabular}


Table 3 - $\quad$ Normal strength of the eight different bond arrangements.

\begin{tabular}{cccc}
\hline & Specimen & \multicolumn{2}{c}{ Normal strength } \\
\cline { 3 - 4 } sarameter & G100RS & Average $\left[\mathrm{N} / \mathrm{mm}^{2}\right]$ & CoV (\%) \\
\hline Bond length & G150RS ${ }^{(1)}$ & 121 & 12 \\
& G200RS & 1318 & 20 \\
\hline $\begin{array}{c}\text { FRP material } \\
\text { Anchor }\end{array}$ & C150RS & 1482 & 11 \\
\hline $\begin{array}{c}\text { scheme } \\
\text { Gubstrate }\end{array}$ & G150RI & 1614 & 16 \\
\hline shape & G150ES & 1316 & 13 \\
\hline
\end{tabular}

${ }^{(1)}$ reference or standard series 